\title{
ChemComm
}

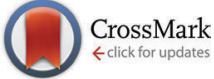

Cite this: Chem. Commun., 2015, 51,7379

Received 13th February 2015 Accepted 18th March 2015

DOI: $10.1039 / c 5 c c 01387 a$

www.rsc.org/chemcomm

\section{DNA polymerase-catalyzed incorporation of nucleotides modified with a G-quadruplex-derived DNAzyme $\dagger$}

\author{
D. Verga, M. Welter, A.-L. Steck and A. Marx*
}

\begin{abstract}
We show that nucleotides which are modified with a G-quadruplexderived DNAzyme are substrates for DNA polymerases. Based on this finding we developed a naked-eye detection system that allows the detection of single nucleotide variations in DNA.
\end{abstract}

Among the entirety of the human genome, single nucleotide polymorphisms (SNPs) are the most common variations. ${ }^{1}$ Between some of these dissimilarities and certain diseases, as well as direct effects of drugs on different patients, exists a direct linkage, a topic dealt with in pharmacogenomics. ${ }^{1,2}$ For this reason, the development of methods that allow time- and cost-effective verification of distinct nucleotide variations in daily laboratory practice is important for the advance in personalized medicine. ${ }^{3}$ Several assays were developed that allow the detection of single nucleotide variations during PCR from genomic DNA samples. Despite PCR-based technologies are broadly applied, they have the inherent disadvantage of requiring temperature cycling using sophisticated instrumentation. These disadvantages make PCR inappropriate for device portability, e.g. for point-of-care testing (POCT) or the detection of pathogens in the field. ${ }^{4}$ To date, several naked-eye detection strategies have been exploited to identify single nucleotide variations and microbial pathogens, including urease/DNAzyme coupled system, ${ }^{5}$ G-quadruplex DNAzyme biosensors, ${ }^{6}$ and DNA probe-modified gold nanoparticles. ${ }^{7}$ However, methods that link single nucleotide incorporation to a signal that is detectable by naked-eye are missing.

Herein, we report a naked-eye detection system that converts the sequence-selective incorporation of a modified nucleotide into a colour change. The method is based on the introduction of a modified nucleotide that contains a G-quadruplex-derived DNAzyme by a DNA polymerase-mediated reaction. The approach is based on our recent finding that DNA polymerases are able to incorporate nucleotides that are modified with unstructured single

Department of Chemistry and Konstanz Research School Chemical Biology, University of Konstanz, Universitätsstrasse 10, 78457 Konstanz, Germany.

E-mail: andreas.marx@uni-konstanz.de

$\dagger$ Electronic supplementary information (ESI) available: Synthetic details for G4-modified dTTP and experimental details for PEX, competitions experiments, solid support reaction, and buffer solution used. See DOI: 10.1039/c5cc01387a stranded oligonucleotides. ${ }^{8}$ Here we show that even nucleotides that bear a structured oligonucleotide "cargo" are sequence-selectively incorporated by DNA polymerases. Since the modification is a signaling unit, the sequence-selective incorporation can be coupled to a signal that is detectable by the naked eye.

Here we exploited the well-established hemin/G-quadruplex DNAzyme sensing probe. ${ }^{9}$ This complex catalyzes the $\mathrm{H}_{2} \mathrm{O}_{2}$-mediated oxidation of $\mathrm{ABTS}^{2-}$ to the correspondent radical $\mathrm{ABTS}^{\bullet-}$ which lead to a readily measurable chromogenic signal. ${ }^{9 a, 10}$

Our approach for the development of a DNA sequence selective naked-eye detection system is depicted in Fig. 1. It is based on performing primer extensions using a biotinylated primer immobilized on streptavidin-coated beads. Upon hybridisation to the appropriate target DNA template a DNAzyme-modified nucleotide will be sequence selectively incorporated. After washing steps, the addition of hemin, $\mathrm{ABTS}^{2-}$, and $\mathrm{H}_{2} \mathrm{O}_{2}$ will induce the formation of the biosensor with green colour generation.

In order to build the depicted system, we first tethered the appropriate 25-nt long oligonucleotide that forms the DNAzyme to a nucleoside- $5^{\prime} O$-triphosphate following the approach we reported recently. ${ }^{8}$ We chose to functionalize the C5 position of the dUTP to introduce the DNAzyme at the nucleobase (Fig. 1B and ESI, $\dagger$ Fig. S1), since it has been shown that several modifications at this position are tolerated by DNA polymerases. ${ }^{11}$

In order to investigate whether the DNAzyme-modified dTTP (1) is processed by DNA polymerases, we examined the acceptance of $\mathbf{1}$ in DNA polymerase promoted primer-extension reactions (Fig. 2A for $K F$ exo ${ }^{-}$DNA polymerase, ESI, $\dagger$ Fig. S2 for KlenTaq DNA polymerase).

We used a 21-nucleotide (nt) ${ }^{32} \mathrm{P}$-labeled 21-nt primer and a 37-nt template (for sequences see ESI $\dagger$ ), which contains a single A residue at position 22, calling for insertion of a thymidine analogue (Fig. 2A). Incubation with $\mathrm{KF} \mathrm{exo}^{-}$DNA polymerase in absence of a thymidine analogue resulted in a primer elongation that is predominantly paused at position 22 without generating significant amounts of full-length product (Fig. 2A, lane 1), while the reaction including all four natural deoxynucleosides triphosphate (dNTPs) showed full-length product (Fig. 2A, line 2). By substitution 


$$
\text { A) }
$$

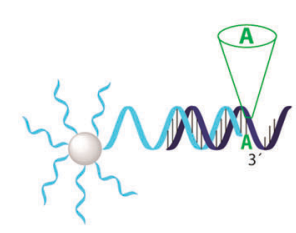

or

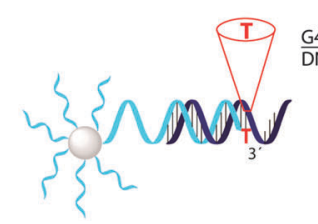

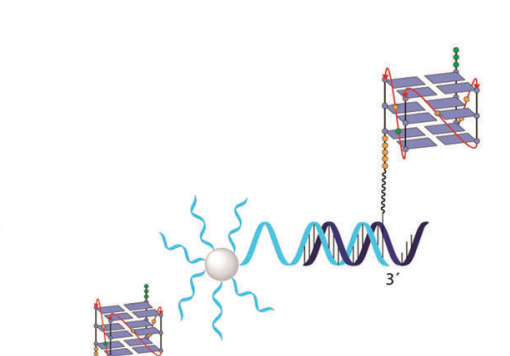

or

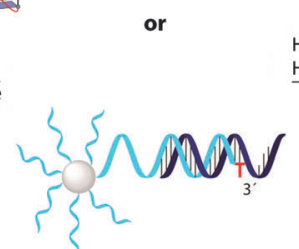

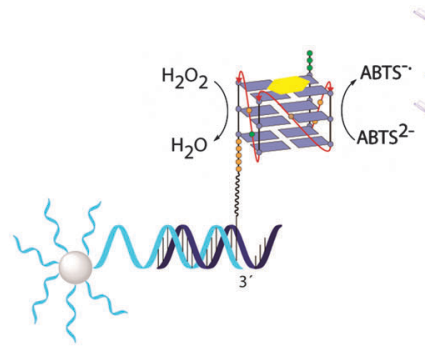

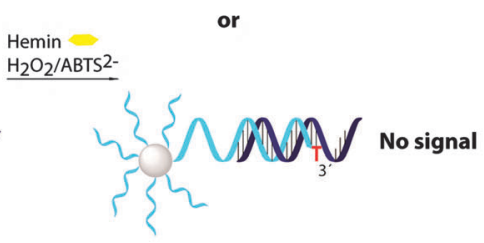

B)

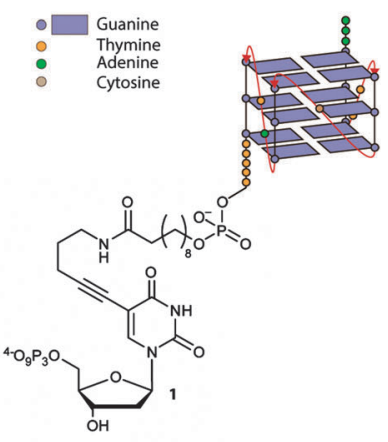

Fig. 1 Concept of coupling sequence-selective nucleotide incorporation to a signal that is detectable by naked eye. (A) Biotinylated primers are immobilized on streptavidin-coated beads. Upon hybridisation of the primer to the appropriate target DNA template a DNAzyme-modified nucleotide is sequence selectively incorporated. After washing steps, the addition of hemin, ABTS ${ }^{2-}$, and $\mathrm{H}_{2} \mathrm{O}_{2}$ will induce the formation of the biosensor with green colour generation in cases where the modified nucleotide was incorporated. (B) Schematic depiction of the DNAzyme-modified dTTP (1).
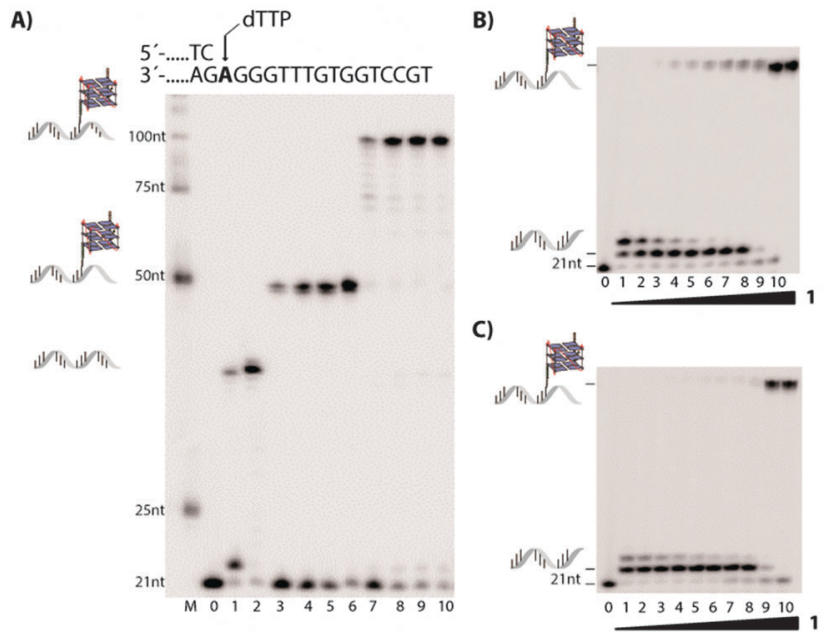

Fig. 2 (A) Partial DNA sequence of primer and template (see ESI† for more details) and PAGE analysis of the primer-extension studies using $K F$ exo ${ }^{-}$DNA polymerase, a 21-nt primer (2), a 37-nt template (3), and $10 \mu \mathrm{M}$ dNTPs. M: DNA marker; lane 0: $5^{\prime}{ }^{32} \mathrm{P}$-labeled primer only; lane 1: primer extension performed in the presence of dATP, dCTP, dGTP; lane 2: same as line 1, but in the presence of dTTP; lanes 3, 4, 5, 6: single-nucleotide incorporation experiments in the presence 1 quenched after 1, 5, 10, and 30 min of incubation; lanes 7, 8, 9, 10: same as lane 1 , but in the presence of 1 , the incorporation reaction was quenched after 1, 5, 10, and 30 min of incubation. (B) PAGE analysis of an exemplary competition experiment employing $\mathrm{KF} \mathrm{exo}^{-}$DNA polymerase $(30 \mathrm{~min})$. The ratio [G4-mod. dTTP (1)]/[dTTP] was varied. Lane 0: 5'-32P-labeled primer only; lane 1: ratio: 0/1; lane 2: ratio 1/10; lane 3: ratio: 1/4; lane 4: ratio: 1/2; lane 5: ratio: 1/1; lane 6: ratio: 2/1; lane 7: ratio: 4/1; lane 8: ratio: 10/1; lane 9: ratio: 100/1, lane 10: ratio: 1/0. (C) PAGE analysis of an exemplary competition experiment employing KlenTaq DNA polymerase (30 min). The ratio [G4-mod. dTTP (1)]/[dTTP] was varied. Lane 0 : 5' - ${ }^{32}$ P-labeled primer only; lane 1 : ratio: 0/1; lane 2: ratio 1/10; lane 3: ratio: 1/4; lane 4: ratio: 1/2; lane 5: ratio: 1/1; lane 6: ratio: 2/1; lane 7: ratio: 4/1; lane 8: ratio: 10/1; lane 9: ratio: 100/1, lane 10: ratio: 1/0.

of the natural thymidine with the DNAzyme-modified nucleoside triphosphate (1) single-nucleotide incorporation product (Fig. 2A, lanes 3-6) and full-length product (Fig. 2A, lane 7-10) were obtained, respectively. As expected, these modified full- length products migrated significantly slower in denaturing polyacrylamide gel electrophoresis (PAGE) than the unmodified full-length reaction product, indicating that the bulky nucleotide was incorporated. Double bands were observed arising from the nontemplated nucleotide addition to the $3^{\prime}$-termini of the blunt-ended DNA strand, as it was previously reported using $3^{\prime}-5^{\prime}$ exonucleasedeficient DNA polymerases. ${ }^{8,12}$ These experiments clearly show that $\mathbf{1}$ is a substrate for the tested DNA polymerases.

To evaluate the efficiency of incorporation of the G4-modified dTTP (1) in comparison to the natural nucleotide we conducted single-nucleotide incorporation experiments in which the modified nucleotide directly competes for incorporation with the natural equivalent (Fig. 2B and C, ESI, $\dagger$ Fig. S3). The ratio of unmodified $v s$. modified nucleotide was determined by PAGE through the significantly different migration caused by the incorporation of the bulky modification. This approach was already utilized for the same purpose as well as to study DNA polymerase selectivity. ${ }^{13}$ We observed that $K F$ exo ${ }^{-}$DNA polymerase incorporates the modified nucleotide with approximately 14 -fold lower efficiency than the natural nucleotide, while we found for KlenTaq DNA polymerase a 22-fold lower efficiency compared to the natural counterpart. The data matched well the previous findings on other C5-modified dTTP analogues. ${ }^{13}$

Encouraged by these results, we went one step ahead and investigated the potential of this approach for DNA analysis. The experiments were done in the sequence context of the B type Raf kinase (BRAF) gene. This gene is a widely known tumor marker, where in a previous study one single-nucleotide substitution was detected in the exon 15: T1796A leading to a substitution of valine by glutamic acid at position 599 (V599E) in the melanoma cell line Colo-829. ${ }^{14}$ For the naked-eye detection system of single nucleotide substitutions, a biotinylated primer strand (for sequences see ESI $\dagger$ ) was immobilized on streptavidin sepharose beads. First, single incorporation experiments of 1 were performed by using the canonical template (for sequences see ESI $\dagger$ ) bearing a dA calling for the incorporation of a dTMP analogue (see Fig. 1A). The results 


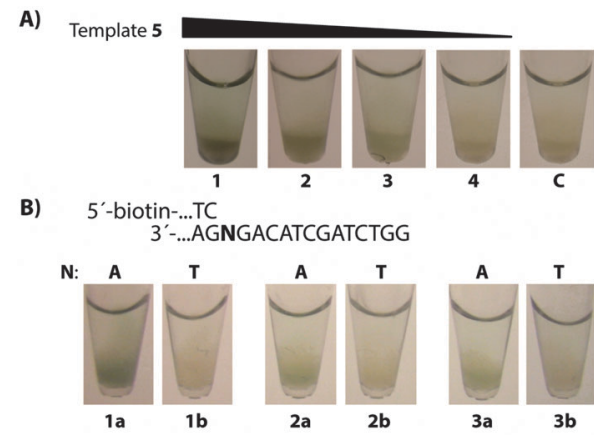

Fig. 3 (A) Identification of the lower detection limit by incorporation of 1 with $K F$ exo- DNA polymerase at $37{ }^{\circ} \mathrm{C}$ in the presence of different concentrations of canonical template. Signal detection via peroxidaselike activity of the incorporated hemin/G-quadruplex DNAzyme. Concentration canonical template: test tube 1: $400 \mathrm{nM}$; test tube 2: $100 \mathrm{nM}$; test tube 3: $50 \mathrm{nM}$; test tube 4: $10 \mathrm{nM}$. Control experiment: test tube C: $400 \mathrm{nM}$ canonical template, experiment performed without 1 . The pictures were taken 45 min after addition of $\mathrm{H}_{2} \mathrm{O}_{2}$. (B) Partial DNA sequence of primer and template (see $\mathrm{ESI} \dagger$ for more details). Discrimination experiments: canonical/non-canonical template. Canonical template concentrations: test tubes 1a (400 nM), 2a (100 nM), and 3a (50 nM); non-canonical template: test tubes $\mathbf{1 b}(400 \mathrm{nM}), \mathbf{2} \mathbf{b}(100 \mathrm{nM})$, and $\mathbf{3 b}(50 \mathrm{nM})$. The pictures were taken 45 min after addition of $\mathrm{H}_{2} \mathrm{O}_{2}$.

are depicted in Fig. 3A. The beads were incubated at $37{ }^{\circ} \mathrm{C}$ for 30 min with $K F$ exo ${ }^{-}$DNA polymerase, 1 and the canonical template. Next, the beads were washed thoroughly and subsequently hemin, ABTS $^{2-}$, and $\mathrm{H}_{2} \mathrm{O}_{2}$ were added to the reaction mixture in an appropriate buffer. The colour generation was visible 20 minutes after the addition of $\mathrm{H}_{2} \mathrm{O}_{2}$, but a better contrast between the different concentrations was observed after 45 min (Fig. 3A, Fig. S4 absorption at $420 \mathrm{~nm}, \mathrm{ESI} \dagger)$. As negative control a reaction without 1 was performed, and no significant colour change was detected. We found a detection limit of about $50 \mathrm{nM}$ (Fig. 3A, 3).

Next we evaluated if the method is able to discriminate, at low concentration, between the presence and the absence of the single nucleotide variation. The experiment was accomplished as described above either in the presence of the canonical template or the non-canonical template (Fig. 3B). We found no significant colour change in the presence of the noncanonical template, even at the lower concentration of $50 \mathrm{nM}$ (Fig. 3B).

In summary, we developed a naked-eye detection system that holds the potential to analyze single nucleotide variations within nucleic acids. The system exploits the sequence selective extension of an immobilized primer by a DNA polymerase using a DNAzyme-modified oligonucleotide. Due to the sequence selective incorporation of the DNAzyme and the subsequent signal, the detection of single nucleotide variations in the nucleic acid analyte is enabled by the naked eye. Here we provide the proof-of-concept that indeed the difference of a single nucleotide variations in the nucleic acid analyte can be transformed into a signal that is read out by the naked eye.

We gratefully acknowledge Alexander von Humboldt Foundation, funding by the DFG and the Konstanz Research School Chemical Biology.

\section{Notes and references}

1 (a) L. Licinio and M. Wong, Pharmacogenomics, Wiley-VCH Verlag GmbH \& Co. KGaA, 2003, pp. i-xl; (b) J. J. McCarthy and R. Hilfiker, Nat. Biotechnol., 2000, 18, 505.

2 (a) A. Crawford, R. G. Fassett, D. P. Geraghty, D. A. Kunde, M. J. Ball, I. K. Robertson and J. S. Coombes, Gene, 2012, 501, 89; (b) R. Nielsen, J. S. Paul, A. Albrechtsen and Y. S. Song, Nat. Rev. Genet., 2011, 12, 443.

3 (a) K. Nakatani, ChemBioChem, 2004, 5, 1623; (b) B. W. Kirk, M. Feinsod, R. Favis, R. M. Kliman and F. Barany, Nucleic Acids Res., 2002, 30, 3295.

4 (a) S. C. Andras, J. B. Power, E. Cocking and M. Davey, Mol. Biotechnol., 2001, 19, 29; (b) Y.-J. Jeong, K. Park and D.-E. Kim, Cell. Mol. Life Sci., 2009, 66, 3325.

5 K. Tram, P. Kanda, B. J. Salena, S. Huan and Y. Li, Angew. Chem., Int. Ed., 2014, 53, 12799.

6 (a) L. Tang, Y. Liu, M. M. Ali, D. K. Kang, W. Zhao and J. Li, Anal. Chem., 2012, 84, 4711; (b) J. Dong, X. Cui, Y. Deng and Z. Tang, Biosens. Bioelectron., 2012, 38, 258.

7 (a) S. Wu, P. Liang, H. Yu, X. Xu, Y. Liu, X. Lou and Y. Xiao, Anal. Chem., 2014, 86, 3461; (b) Y. Akiyama, H. Shikagawa, N. Kanayama, T. Takarada and M. Maeda, Chem. - Eur. J., 2014, 20, 17420.

8 A. Baccaro, A.-L. Steck and A. Marx, Angew. Chem., Int. Ed., 2012, 51, 254.

9 (a) P. Travascio, Y. Li and D. Sen, Chem. Biol., 1998, 5, 505; (b) Y. Li and D. Sen, Biochemistry, 1997, 36, 5589.

10 (a) F. Du and Z. Tang, ChemBioChem, 2011, 12, 43; (b) R. Freeman, E. Sharon, C. Teller, A. Henning, Y. Tzfati and I. Willner, ChemBioChem, 2010, 11, 2362; (c) D. Li, B. Shlyahovsky, J. Elbaz and I. Willner, J. Am. Chem. Soc., 2007, 129, 5804; (d) Y. Xiao, V. Pavlov, T. Niazov, A. Dishon, M. Kotler and I. Willner, J. Am. Chem. Soc., 2004, 126, 7430.

11 (a) S. H. Weisbrod and A. Marx, Chem. Commun., 2008, 5675; (b) M. Hocek, J. Org. Chem., 2014, 79, 9914.

12 (a) S. Obeid, N. Blatter, R. Kranaster, A. Schnur, K. Diederichs, W. Welte and A. Marx, EMBO J., 2010, 29, 1738; (b) J. M. Clark, Nucleic Acids Res., 1988, 16, 9677; (c) S. Obeid, A. Schnur, C. Gloeckner, N. Blatter, W. Welte, K. Diederichs and A. Marx, ChemBioChem, 2011, 12, 1574.

13 J. G. Bertram, K. Oertell, J. Petruska and M. F. Goodman, Biochemistry, 2009, 49, 20.

14 H. Davies, et al., Nature, 2002, 417, 949. 